\title{
EL MÉTODO DE CASO COMO ESTRATEGIA DIDÁCTICA APLICADA A LA ENSEÑANZA DE LA GESTIÓN DE LA INFORMACIÓN EN LA UNIVERSIDAD NACIONAL DE LA PLATA, ARGENTINA
}

María Cecilia Corda

Universidad Nacional de La Plata (UNLP)

Argentina

Marcela Karina Coria Universidad Nacional de La Plata (UNLP) Argentina

Ana Ayala

Universidad Nacional de La Plata (UNLP)

Argentina

Rafaela Stefanizzi

Universidad Nacional de La Plata (UNLP)

Argentina

Universidade Nacional de La Plata; Argentina.

\section{RESUMEN}

El presente trabajo aborda el 'Método de Caso' como estrategia didáctica, diferenciándolo del método 'Estudio de Caso' según queda aclarada la conceptualización de ambos en la bibliografía recopilada. Asimismo, se analiza la experiencia desarrollada respecto al mencionado método en el marco de la cátedra 'Gestión de la Información', de la carrera de bibliotecología y ciencia de la información de la Universidad Nacional de La Plata (Argentina). Se efectúa un balance de esta aplicación a lo largo de más de 10 años y se hace una recomendación respecto a su utilidad en instancia de formación universitaria de grado.

Palabras- Clave: Método de Caso; Prácticas Pedagógicas; Enseñanza de la Bibliotecología, Universidad Nacional de La Plata; Argentina.

Palavras-Chave: Método de Caso; Práticas Pedagógicas; Ensino de Biblioteconomia; 


\section{THE CASE METHOD LIKE A \\ PEDAGOGIC STRATEGY IN \\ INFORMATION MANAGEMENT \\ EDUCATION AT UNIVERSIDAD NACIONAL DE LA PLATA, ARGENTINA}

\section{ABSTRACT}

The present paper aims to the case method as didactic strategy, separating it from the case study according to the conceptualization of both in the compiled bibliography. In the same way, there analyzes the experience developed with the mentioned method in the context of the chair Management of the information, of the career of Library and Information Science of the National University of La Plata (Argentina). There is a balance of this application throughout more than 10 years and a recommendation about his usefulness in the higher education.

Keywords: Case Method; Library Sciences Teaching; Universidad Nacional de La Plata; Argentina.

\section{INTRODUCCIÓN}

El plan de estudios vigente en el Departamento de Bibliotecología de la Universidad Nacional de la Plata (UNLP) data del año 2004. Esta propuesta mantuvo los tres títulos de grado otorgados por el plan anterior de 1986, aunque sus nombres variaron: de Bibliotecario Documentalista a Bibliotecólogo, y de Licenciado o Profesor en Bibliotecología y Documentación a Licenciado o Profesor en Bibliotecología y Ciencia de la Información. Con excepción de las asignaturas Tratamiento de la información I y II e Historia del libro y de las bibliotecas, las materias restantes sufrieron modificaciones en sus denominaciones (LAUDANO; PLANAS; CORDA, 2011).

En el mencionado plan se incorporaron nuevas asignaturas, entre ellas, "Política de la Información"; "Usuarios de Información”; mientras que "Gestión de la Información" vino a convertirse en la equivalencia de una materia anterior llamada "Documentación General". Esta última se ubica en el tercer año de la carrera de Licenciatura o Profesorado en Bibliotecología y Ciencia de la Información y tiene régimen cuatrimestral. La misma reviste carácter de asignatura integradora, ya que articula muchos de los conceptos, las metodologías y las prácticas correspondientes a las materias que la preceden en el plan. Además, es novedosa porque desarrolla tendencias avanzadas en el campo profesional, pretende estimular la reflexión teórica y el análisis de experiencias para una formación acorde con las necesidades de las organizaciones donde el alumno o la alumna puedan insertarse 
laboralmente en el futuro una vez que egresen.

En el presente trabajo, se analiza cómo la aplicación del método de caso ha resultado ser una estrategia didáctica de utilidad para la enseñanza de la mencionada asignatura. Se presentan sucintamente, desde el punto de vista conceptual, las diferencias con el estudio de caso, más ligado a la esfera de la investigación. Se repasan también los lineamientos esenciales para su comprensión y aplicación pedagógica. Finalmente, se reflexiona sobre su utilización en el marco de la citada cátedra a lo largo de más de diez años.

Cabe destacar que los casos, en tanto estrategias de enseñanza, son una forma de proporcionar campos de práctica en la formación del estudiante, y también del docente, porque sitúan al estudiante en una serie de problemas e incluyen prácticas que encontrarán en el mundo real. Lo que los prepara para la resolución de auténticos problemas y permite el desarrollo del pensamiento crítico. En este sentido, Argandoña (1999, p.6) agrupa los métodos de enseñanza en activos (que transmiten conocimientos vía comunicación) y pasivos (vía descubrimiento), aunque los límites entre unos y otros no están bien definidos. La gama va desde las clases magistrales, conferencias y lecturas, pasando por las discusiones en clase o en seminario, la resolución de ejercicios, la elaboración de informes (escritos $\mathrm{u}$ orales), la simulación, la discusión de problemas (reales o ficticios), mini-casos, ejemplos etc., hasta métodos más participativos, como el método del caso, el "role playing", los trabajos de campo y las prácticas.

El método en sí es factible de ser aplicado en múltiples materias impartidas en la enseñanza de la bibliotecología, tales como gestión de unidades de información, ética de la información, derecho a la información, políticas de información, estudios de usuarios, entre otras.

El recurso es también utilizado con frecuencia para resolver los dilemas éticos que se pueden presentar en el ejercicio de la profesión. Por lo que también es una técnica recomendada para la enseñanza de la ética, ya que reviste suma utilidad como estrategia didáctica para trabajar con aquellos estudiantes que tienen diversos niveles de formación, morales y/o profesionales respecto a asuntos de relevancia en el campo disciplinar. A propósito, además del trabajo de Argandoña (1999) en el 
cual profundiza sobre la enseñanza de la ética con la implementación del método de caso, se destaca el de Vargas Vasserot (2009), quien propone una experiencia de aplicación de este mismo método para el aprendizaje en el contexto de una cátedra de la carrera de derecho en la Universidad de Almería (España).

A continuación, se efectúa un análisis bibliográfico para dilucidar conceptualmente entre método y estudio de caso de acuerdo a las discusiones plasmadas en el material especializado al que se tuvo acceso.

\section{MÉTODO DE CASO Y ESTUDIO DE} CASO

Los casos, señala Bonache (1999), suelen utilizarse como recurso pedagógico para aproximar la realidad organizacional de empresas o instituciones a las aulas, haciendo que los debates y las explicaciones se aproximen lo máximo posible a la misma. Remarca también que este uso didáctico está muy extendido, así como también los casos se emplean como estrategia de investigación.

Aunque es un dilema de vieja data, el intercambio de denominaciones entre la esfera didáctica y la investigativa aún subsiste en el campo pedagógico: método de caso y estudio de caso. Esto se puede ver en documentos de instituciones que fueron precursoras y lo adoptaron como parte de su sistema de enseñanza, tal es el ejemplo del Instituto Tecnológico y de Estudios Superiores de Monterrey. Inclusive, en producciones recientes en nuestro campo como la de González Pérez (2015), se continúa usando la denominación estudio de caso para referirse a la estrategia de enseñanza.

A fin de aclarar la mencionada disyuntiva, se puede recurrir al trabajo de Miglioli (2014), quien brinda una breve y concisa definición sobre uno y otro: el método de casos está vinculado a una metodología de enseñanza cuyo objetivo es permitir al alumno o la alumna tomar decisiones a partir de una situación que puede darse en el mundo real (MIGLIOLI, 2014, p.5). En cambio, estudio de casos se usa para referirse a la metodología científica en la cual la descripción del caso es precedida por una discusión teórica sobre los temas que se desean abordar, lo que confirma o no los supuestos teóricos sostenidos e implica el análisis en profundidad de un fenómeno o hecho (MIGLIOLI, 2014, p.5). 


\section{Rebecin \\ REVISTABRASILEIRAde
EDUCAÇÃO em CIÊNCIA da INFORMAÇÃO}

Entonces, el método de caso es un tipo de discusión interactiva que emplea la herramienta educativa llamada caso.

Los casos son "[...] instrumentos educativos complejos que revisten la forma de narrativas" (WASSERMANN, 2006, p.19).

César (2005) también aporta claridad conceptual en relación a la aplicación del caso, explicando que en la faz investigativa se trata de un método cualitativo $y$, en la faz didáctica, de un método de enseñanza. Afirma que el método de caso no se encuentra tan distante del método de estudio de caso en el marco de la investigación científica. Ambos precisan ser construidos a partir de evidencias recolectadas sobre una realidad $y$ persiguen propósitos definidos a partir de una base teórico-conceptual. No obstante, también aclara, que en el primero se adoptan ciertas "licencias poéticas", dado que a veces es necesario adaptar el caso para conseguir los fines didácticos. Hay que puntualizar que este autor tiende más a tomar casos reales que ficticios, situación que no siempre es posible en el ámbito de la bibliotecología dado que no existe en lengua castellana una tradición de escritura de los casos en ningún formato (artículos, presentaciones a congresos, notas etc.).

Asimismo, advierte sobre cuestiones éticas ligadas a su implementación en la enseñanza y su aplicabilidad de acuerdo al momento y contexto de la enseñanza de determinada disciplina, con posibilidades de combinación con otros métodos didácticos.

\section{CARACTERÍSTICAS DEL MÉTODO DE CASO}

El método de caso, dentro de la concepción explicada por Estrada Cuzcano y Alfaro Mendives (2015, p.198) es tanto una técnica como una metodología educacional que pone a consideración del estudiantado situaciones y problemas verdaderos que conducen a la presentación de alternativas, soluciones y resoluciones. Se puede decir que este método es un tipo de discusión interactiva que está estructurado sobre la base del estudio de casos y el cual es referido comúnmente con el término caso. Según García del Junco y Castellanos Verdugo (1998, p.97), el concepto de caso refiere a de la "[...] descripción de una situación auténtica donde se 


\section{Rebecin \\ REVISTABRASILEIRAde
EDUCAÇÃO em CIÊNCIA da INFORMAÇÃO}

presenta o puede presentarse uno o varios problemas con toda su complejidad y riqueza de matices tal cual es en la vida real". En tanto, su contenido puede exhibirse en una exposición oral $\mathrm{o}$ en cualquier otro soporte impreso y/o audiovisual.

El principal objetivo del método de caso es desarrollar en el alumnado las capacidades para la resolución de problemas complejos y la toma de decisiones en situación de ambigüedad, incertidumbre y presión (aunque sin los riesgos de la toma real de decisiones). Esas capacidades incluyen el análisis de situaciones complejas, la elaboración de juicios sobre ellas (definición de problemas), la toma de decisiones, la apreciación de las consecuencias de las mismas, y la comunicación (ARGANDOÑA, 1999, p.8). A partir de éste, se desprenden otros objetivos, igualmente relevantes de acuerdo al autor:

- La aplicación de teorías y técnicas al análisis de temas reales. Esto se puede conseguir también mediante ejercicios, problemas abstractos, "mini-casos" etc.

- El fomento de la comunicación entre quienes participan.
- El incentivo al interés del alumno, haciéndolo el primer responsable de su propia formación.

- El diseño de planes de acción razonables, consistentes y creativos.

- El reconocimiento de la significación de la información.

El método de caso tiene correlación técnica y metodológica a la vez, y se aplica con éxito en muchas disciplinas universitarias y en diversas actividades académicas. La enseñanza a través de este método siempre supone diferencias y diversos puntos de vista, y requiere cumplir con algunas condiciones relacionadas con la forma y el estilo necesarios para su eficaz desarrollo en el aula (ESTRADA CUZCANO; ALFARO MENDIVES, 2015, p.197).

Este recurso resulta interactivo entre estudiantes y docente, ya que los conocimientos se construyen paso a paso, lo que posibilita la comprensión por parte de los alumnos de la estructura global que sustenta una situación real que puede reclamar un diagnóstico ante lo que se plantea (GARCÍA DEL JUNCO; CASTELLANOS VERDUGO, 1998, p.97). 


\section{Rebecin \\ REVISTABRASILEIRAde
EDUCAÇÃO em CIÊNCIA da INFORMAÇÃO}

Además, es útil para la resolución de problemas no operativos, en los que la referencia a teorías y técnicas es necesaria, pero no agota el proceso de análisis y decisión. La pedagogía del método del caso se basa en aprender haciendo, en un proceso ordenado, bajo la guía del profesor o de la profesora, utilizando la "experiencia directa guiada" (una experiencia simulada, pero basada en situaciones reales) para crear hábitos de resolución de problemas no operativos. El caso descrito se convierte en la clase en un caso "vivo", una realidad intelectual y emocional, en que el alumnado se involucra como decisor, de ahí su atractivo y su potencialidad motivadora (ARGANDOÑA, 1999, p.7).

El método de caso puede considerarse una técnica de simulación en la clase, aunque previamente el docente o la docente debe planificar el método según las características del entorno, los destinatarios y la complejidad del asunto tratado (ESTRADA CUZCANO; ALFARO MENDIVES, 2015, p.197).

La clave para la comprensión del método de enseñanza basado en casos se encuentra en el posicionamiento del alumno dentro de una situación real, dándole oportunidad de dramatizar sus propias decisiones $y$, por ende, preparándolo para la acción. Esto lleva a la conclusión de que la participación de las personas es indispensable para la utilidad del método. De esta manera, la clase se conduce mediante un liderazgo participativo en contraposición con el método clásico que está exclusivamente regido por la figura del docente. Un valioso corolario de esta metodología es la práctica que da al estudiante en lo que respecta al proceso comunicativo de escucha, comprensión e intercambio de ideas.

Precisamente, García del Junco y Castellanos Verdugo (1998, p.100) sostienen que la aplicación de este método permite una formación individual, ayudando al alumnado a pensar y manejar prioridades y conceptos que asimilan en su formación. El espíritu crítico se va potenciando en situaciones determinadas, a la vez que las ideas toman un cauce práctico. De esta forma, el grado de comunicación mejora, como así también, el aprendizaje para relacionarse con los demás y exponer los propios pensamientos. En síntesis, se agudiza y desarrolla la capacidad para la reflexión y la toma de decisiones. 
Este método, aplicado principalmente en la enseñanza universitaria, le proporciona también otros beneficios a quien está en formación como profesional:

- Entendimiento e interpretación de los datos.

- Reconocimiento de suposiciones e inferencias opuestas a lo concreto.

- Pensamiento analítico y crítico.

- Evaluación de las relaciones interpersonales.

- Ejercitación y elaboración de juicios.

- Comunicación de ideas y opiniones.

- Toma y defensa argumental de decisiones.

Asimismo, el recurso proporciona una experiencia sujeta a la realidad y es muy versátil ya que permite aplicar proposiciones teóricas y conjugarlas con simulaciones prácticas, además de estimular la creatividad y toma de decisiones. Del mismo modo, se orienta a la flexibilidad y la apertura, fomenta la discusión concienzuda del problema de estudio y propicia el intercambio de ideas, evitando la creación de un medio estático.
Los ya citados Estrada Cuzcano y Alfaro Mendives (2015, p.197) postulan que este método es adecuado para la enseñanza universitaria debido a que no está basado sólo en la intervención o los conocimientos del docente, sino fundamentalmente en la participación activa del estudiante, quien identifica, analiza, sistematiza, evalúa, propone y decide cómo resolver las situaciones o problemas planteados en el caso. En este contexto, el profesor o la profesora desempeñan un rol fundamental, ya que son responsables del diseño del caso, la preparación de la asignatura, la elección de la pedagogía empleada y la conducción de la clase. La enseñanza a través del método de caso es continua, en la cual el docente debe cumplir varios roles: puede ser absorbido como iniciador, director, participante y motivador, aunque adicionalmente se podría añadir el rol de mediador o facilitador (ESTRADA CUZCANO; ALFARO MENDIVES, 2015, p.200).

Argandoña (1999, p.10) asegura que el éxito del método del caso depende, en buena medida, del profesor o de la profesora, quien debe mostrarse "neutral" (aunque resulte utópico plantearlo) y receptivo/a ante las diferentes opiniones expresadas por sus 
estudiantes. Esto es, le corresponde tomar las preconcepciones, las experiencias y los sentimientos del grupo de estudiantes, ayudar a explicitarlos, analizarlos críticamente, y conducir el diálogo de tal modo que pueda modificar libremente su punto de vista mediante argumentos racionales. En este sentido, se deben evitar prácticas tales como la alusión a un punto de vista como argumento descalificador del resto de la clase, el predominio de un criterio en particular, la conducción de la discusión de forma que salga triunfante una tesis, la limitación de opiniones discrepantes y, en general, todo lo que pueda provocar la inhibición del alumnado.

En todo caso, lo que importa no es que se conozca la "conclusión correcta" del caso, sino los principios, y desarrollen sus capacidades de análisis, decisión y su conciencia ética. En resumen, quien está a cargo de la clase debe tener la preocupación más por el proceso de aprendizaje que porque se llegue colectivamente a la "decisión correcta" del caso, sin perjuicio de que, si la hay, la señale (ARGANDOÑA, 1999, p.11).

Así, el método de casos busca la flexibilidad y la apertura, fomenta la discusión a conciencia del problema de estudio y propicia el intercambio de ideas.

El caso debe incluir información y datos variados de índole psicológicos, sociológicos, antropológicos, históricos y demás, como también material técnico. Aunque se centra en temáticas específicas de acuerdo a la enseñanza del campo del conocimiento, es interdisciplinario. Por lo general, las narrativas del caso se basan en problemas de la vida cotidiana y que se le pueden presentar a personas reales.

\section{CONSIDERACIONES SOBRE LA APLICACIÓN DEL MÉTODO DE CASO}

La aplicación práctica de esta metodología consiste precisamente en la propuesta por parte del docente a sus estudiantes de una serie de casos que representen situaciones problemáticas diversas de la vida real para que se estudien y analicen en la clase (ESTRADA CUZCANO; ALFARO MENDIVES, 2015, p.199).

La presentación del caso requiere de algunas condiciones: realizar una discusión como parte integral del método; utilizar distintas herramientas para dirigir la discusión; recibir contribuciones de pares; considerar una 
gran diversidad de las características individuales de las y los estudiantes; reconocer los casos como incompletos; suponer que no hay una única decisión correcta y que la evidencia que apoya una decisión particular puede provenir de muchas fuentes (ESTRADA CUZCANO; ALFARO MENDIVES, 2015, p.200).

Estos mismos autores proponen el establecimiento de los pasos necesarios para la adopción de buenas prácticas en la aplicación de este método:

- Mejorar el contacto entre estudiantes y docentes.

- Desarrollar reciprocidad y cooperación entre estudiantes.

- Proporcionar retroalimentación inmediata.

- Priorizar el tiempo en la tarea.

- Generar altas expectativas (con respecto a la solución).

- Respetar la diversidad de los talentos y los procesos de aprendizaje.

Los pasos para su aplicación según Estrada Cuzcano y Alfano Mendives (2015) son los siguientes:

1. Preparación del caso: determinación de elementos, antecedentes,

hechos, problemas.

2. Discusión del caso: análisis, diagnóstico, recolección de datos, alternativas de solución, plan de acción.

3. Decisión del caso: solución, recomendación, toma de decisiones.

4. Presentación del caso: sinopsis, conclusión, estructuración del informe.

Además, resaltan que los casos deben cumplir con 6 reglas (ESTRADA CUZCANO; ALFANO MENDIVES, 2015, p.203):

1. Verosimilitud, que sea creíble para quien lo lee;

2. Veracidad, que esté basado en hechos reales;

3. Interés y actualidad, que sea novedoso para el alumnado;

4. Controversia; que genere discusión;

5. Concreción, que describa los hechos incluidos someramente;

6. Comprensión, que brinde los elementos necesarios de contexto y de la situación planteada en sí para que pueda 
analizarse, ya que la complejidad no tiene que ser sinónimo de confusión.

Al final de cada caso se presentarán preguntas críticas, con el objetivo de promover la comprensión, que orientarán al alumnado a examinar ideas importantes, nociones y problemas relacionados con el caso. Por lo tanto, la redacción de estas preguntas requerirá de mucho trabajo por parte del docente, ya que con ellas se espera que el grupo de estudiantes tenga reflexiones inteligentes sobre los problemas y aplique sus conocimientos cuando examina ideas.

Estas preguntas no tienen como propósito que las/los alumnas/os recuerden una información sobre hechos y produzcan respuestas específicas. Tampoco que lleguen a conocer algunos fragmentos de información sobre los hechos o que memoricen nombres, fechas, descripciones etc.

La enseñanza basada en el método de casos permite que los alumnos y las alumnas trabajen en pequeños grupos para discutir las respuestas que darán a las preguntas críticas. El trabajo en los grupos prepara para la discusión que se producirá posteriormente con participación de toda la clase.

Al promover el análisis crítico del alumnado, la o el docente se debe abstener de intercalar sus propios pensamientos a fin de que no influyan en el pensamiento de quienes están tratando el caso ni lo obstaculicen. Por ello, la coordinación requiere gran habilidad en la escucha, además de la capacidad de poder resumir las ideas, reformular preguntas de análisis de las cuestiones medulares, respetar las ideas de todos los alumnos sin juzgarlas, lograr que todos se sientan cómodos para expresarse, encauzar la discusión, además de evitar que se desvíe el tema.

Esta fase de discusión del caso debería generar más preguntas que respuestas, por lo tanto, se espera que las alumnas y los alumnos deseen ampliar la información. A tal fin se pueden utilizar variados recursos, por ejemplo, libros de texto, artículos de diarios y revistas, tablas y gráficos con datos primarios, informes de investigaciones, editoriales y otros comentarios, novelas, películas, tanto comerciales como documentales. Por su parte, las y los estudiantes pueden 


\section{Rebecin \\ RDUCAÇÃO em CIÊNCIA da INFORMAÇÃO
EDSE}

realizar estas actividades de seguimiento en forma individual o colectiva.

Sean cuales fueren las actividades de seguimiento que se elijan, su valor aumentará si se realizan nuevas discusiones como la que acompaña al interrogatorio y se lleva a cabo en ellas un examen ampliado de los problemas, con introducción de nuevas perspectivas. A través de este proceso, el examen reflexivo y crítico que hacen los alumnos de los problemas importantes evoluciona continuamente (WASSERMANN, 2006, p.2728).

En base a lo antedicho, el método presenta algunas complicaciones para quienes poseen dificultades de comunicación o para quienes no responden rápidamente a los problemas presentados. En consecuencia, el método de caso aplicado en la clase tiene un valor fundamental y según la complejidad del mismo puede conducir al estudiante a desarrollar actividades, habilidades, pensamiento crítico e investigación científica (ESTRADA CUZCANO; ALFARO MENDIVES, 2015, p.201).

\section{APLICACIÓN DEL MÉTODO DE CASO EN LA ENSEÑANZA DE LA ASIGNATURA GESTIÓN DE LA INFORMACIÓN}

Los relatos sobre situaciones reales o ficticias sobre las que hay que tomar decisiones haciendo las veces del director/a de una biblioteca, centro de información o documentación; o bien las de jefe/a de algún sector, área o departamento, favorecen el desarrollo de la creatividad y la reflexión sobre situaciones concretas o hipotéticas que pueden darse en los mismos lugares de trabajo de las y los participantes. Sin embargo, hay autores como César (2005) que rechazan la idea del método de caso ligado a una situación ficticia, y agregan que no solo no es un caso algo que refiere a ello, sino que tampoco lo es un ejercicio que presenta datos de una organización, de tipo más descriptivo; ni un material usado como ilustración o ejemplo de alguna hipótesis o concepto.

Los casos a los que se alude en el contexto de la asignatura "Gestión de la Información" son relatos que conllevan tintes de realidad, pero que no están muchas veces referidos a un contexto organizacional dado, ya que ello implicaría, por cuestiones éticas o legales, tener permisos, acceder a información de uso interno sino confidencial, además de, al ser un ambiente más o menos reducido, correr 
el riesgo de suscitar rispideces con colegas que trabajan en los centros de referencia. En algunas ocasiones hay aproximaciones a casos publicados en un artículo académico o ponencia, entonces se pueden emplear para analizar ciertos conceptos 0 determinadas metodologías de la gestión de la información. Aunque, cabe aclarar, suelen ser incompletos dado que no están pensados para la enseñanza.

La estrategia didáctica que se propone en el marco de la asignatura exige realizar el esfuerzo de colocarse en la situación planteada y tomar la decisión más apta para la resolución del problema. Para asegurar su utilidad didáctica (CORDA, 2012), se procura que los casos planteados revistan tres rasgos: por un lado, autenticidad (son relatos sobre situaciones concretas sacadas o referidas a la realidad); por otro, urgencia de la situación (problemáticas que exigen un análisis o una decisión más o menos inminente); y, por último, orientación pedagógica (situaciones que proporcionan información y formación sobre un dominio del conocimiento o de la acción operativa vinculada con la gestión, en la experiencia analizada).
Una variable importante que se incluye en los casos a tratar por los alumnos y las alumnas es la del tiempo. Esto, un poco condicionado por la duración misma de la clase, otro poco por la exigencia de la gestión misma en la vida real, que impulsa a tomar decisiones o cursos de acción en base a las informaciones disponibles y la urgencia de resolución de cuestiones cotidianas.

La mayoría de los casos son, entonces, relatos ficticios basados en realidades posibles en la esfera de bibliotecas, centros de información o documentación. Mayormente, se toma también de base una institución o empresa real para poder extraer información de contexto de, por ejemplo, su sitio web. Se acompañan de preguntas que plantean los dilemas a los que se enfrentan las personas que están involucradas en el caso. Dicha estrategia se orienta, fundamentalmente, a la toma de decisiones que es uno de los pilares esenciales de la gestión.

De forma puntual, en un trabajo práctico se combina el método de caso con el rol playing, es decir, no solamente se analiza el caso y se toman decisiones o cursos de acción respecto a la 
situación planteada, sino que los alumnos y las alumnas asumen el papel de consultores respecto a un proyecto de arquitectura de información para un sitio web institucional que se desea reformular. Esta actividad lleva a que desarrollen una presentación profesional pautada en un rango de tiempo determinado; expongan la propuesta empleando recursos audiovisuales; defiendan su punto de vista y "compitan" con otros equipos por ser seleccionada por los miembros de la institución convocante, representados por las docentes de la cátedra. A continuación, se reproduce el planteo del caso correspondiente a este trabajo práctico.

Universidad Nacional de La Plata Facultad de Humanidades y Ciencias de la Educación

Departamento de Bibliotecología

Cátedra: Gestión de la Información

Tema: Arquitectura de la información

Concurso: "La Universidad del futuro se acerca a nosotros"

La Universidad Villaplata llama a la presentación de proyectos para el diseño de su portal universitario.

¡Presente su propuesta cuanto antes!

\subsection{Contexto General}

La Universidad Villaplata es una institución de educación superior privada que ofrece carreras en 6 áreas del conocimiento (Medicina, Derecho, Humanidades, Ciencias Económicas, Informática, Ciencias Agrarias). Su comunidad académica está conformada por 100 personas que desarrollan tareas administrativas, 2.500 alumnos y 170 docentes.

Este año, la Universidad cumple 25 años, y quiere festejar su aniversario con un portal web rediseñado. Le interesa mostrar que las nuevas tecnologías de información y comunicación, la sociedad de la información, la democratización del conocimiento, el desarrollo mundial no son sólo palabras para ella, sino que es una universidad que apuesta a un futuro tecnológico, democrático y culto.

Para lograr estos objetivos, ha abierto una convocatoria donde se invita a varias empresas a presentar proyectos en los cuales se plantee el diseño de un portal universitario. Este sitio es la entrada principal de la Universidad; por lo que, su objetivo es servir de enlace entre la institución y todos los integrantes de su comunidad que estén interesados en la información que ésta genera y los servicios que ofrece en línea. De esta forma el portal universitario se convierte en la voz 
oficial de la Universidad en medios electrónicos.

El plazo de cierre para la presentación de proyectos es la clase próxima práctica. Ese día, cada empresa podrá presentar su proyecto de arquitectura de información, explicando en qué consiste su propuesta, qué información incluye, cómo está organizada y estructurada, enunciar las ventajas que le proporcionará a la Universidad el tener su sitio web, por qué la Universidad debe elegir ese proyecto en particular, etc.

Una vez finalizadas las presentaciones, un jurado exigente compuesto por miembros de la Universidad decidirá cuál es el mejor proyecto, financiando la implementación del mismo.

\subsection{Indicaciones}

Suponga que usted y su grupo son miembros de una joven empresa comercial que se dedica al diseño de este tipo de proyectos. Vuestro jefe, el Sr. Rodríguez, les ha encomendado la tarea de ganar este concurso. Ustedes tendrán que demostrar toda su profesionalidad elaborando un buen proyecto, interesante, novedoso..., y vendiéndolo de la mejor forma posible. El vendedor de proyectos más hábil será aquél que logre conquistar al jurado universitario.

¿Qué tienen que hacer?:

1) Planificar la arquitectura de información del sitio web. Para ello deberán:

- definir cuáles son los contenidos que incluirían,

- organizar un mapa del sitio donde se visualice claramente la información incluida y su posición/ubicación dentro del sitio.

Puede consultarse la bibliografía indicada para aclarar dudas y tomar ideas.

2) Preparar la presentación oral del proyecto:

Se expondrá oralmente en clase cada proyecto propuesto, explicando en qué consiste la propuesta, qué información incluye, cómo está organizada y estructurada, enunciando las ventajas que le proporcionará a esta casa de estudios contar con su propio sitio web, explicando por qué la Universidad debe elegir ese proyecto en particular etc. 
El objetivo es que se inicien en el ejercicio de "vender" ideas, defender proyectos propios e incursionar en áreas en las que se está acostumbrado a trabajar. Por lo que, esta exposición deberá realizarse como si ustedes fueran vendedores expertos, no como estudiantes, tratando de convencer al jurado de que adquieran su proyecto. Para eso se valdrán de todas las herramientas que utilizan los vendedores para convencer a sus posibles futuros clientes (presentaciones gráficas, carteles, folletos, material audiovisual, argumentaciones orales, establecimiento de vínculos personales etc.).

En otras aplicaciones que se hace del método de caso en la enseñanza de la asignatura en cuestión, se emplean relatos dialogados con actores que pueden encontrarse en la gestión cotidiana de una unidad de información (por ejemplo: directora de la biblioteca, responsable del área de informática, proveedora de servicios informáticos, usuario especializado de información). Así, leyendo en voz alta e interpretando esos diálogos, el alumnado se interioriza de la situación o del dilema planteado y se abre la discusión sobre qué decisión adoptar y los fundamentos que la sostienen en base a los temas que se van desarrollando en la clase. A veces el resultado es el diseño de un plan, como puede ser en el caso usado para el tópico auditoría de la información, situado en una biblioteca universitaria. Otras veces, se trata de la elaboración de un texto en formato de aviso clasificado laboral para un diario, como puede ser en el caso utilizado para abordar el perfil profesional del gestor de la información, situado en una empresa mediana dedicada a la arquitectura y el diseño que requiere un puesto de trabajo a cubrir con competencias y formación en ese sentido. En otras ocasiones, la variable del tiempo juega un papel esencial, y se solicita la ejecución de ciertas actividades en plazos perentorios, para probar la habilidad de cada alumno en resolver la solicitud de una supuesta consultora del área de ciencias de la información respecto a temas de repositorios o bibliotecas digitales.

Respecto a la aplicación del método de caso en el contexto del aula universitaria, se coincide con lo sostenido por Ogliastri (2005), en el sentido que el propósito es aprender a 
pensar y tomar decisiones fundadas, y no tanto llegar a una resolución ideal de la problemática $\mathrm{o}$ al parafraseo de conceptos y metodologías planteadas en la bibliografía de apoyo o sugerida desde la cátedra.

Un caso es la descripción de una situación real, que se discute en clase bajo la dirección de un profesor. Las soluciones de un caso son imperfectas, por lo que no se trata entonces de resolver problemas en grupo para encontrar la solución correcta. [...] En el método de casos importan por igual los contenidos de la materia como el proceso mismo de conocerla; este proceso ayuda a formar en los estudiantes una capacidad de enfrentar situaciones nuevas con criterios propios, pues la responsabilidad del profesor va más allá de transmitir unos conocimientos: se trata de que los estudiantes aprendan a pensar por su cuenta (OGLIASTRI, 2005, p.7).

\section{CONCLUSIONES}

Así como García del Junco y Castellanos Verdugo (1998) proponen el método de caso anexado con el concepto de situación, resultaría interesante probar en próximas cursadas de esta asignatura su funcionamiento. El tipo de metodología que estos autores probaron para sus propias materias del área de la
Administración consiste en recoger un conjunto de problemas, proporcionando una perspectiva sistémica y holística para el alumnado. Este trabajo en equipos conlleva un esfuerzo mayor si tiene que determinar el problema común entre las distintas situaciones que presenta el caso. Así, un caso estará formado por situaciones, cada una de las cuales recoge el conjunto de problemas al que se aludía, en los que se detectará cuál es el común a todas las situaciones que se presentan en un caso. Finalmente, llegan a una solución común a los problemas que se vislumbran o detectan.

Para finalizar, se puede sostener que la aplicación de este tipo de estrategias en la enseñanza de la bibliotecología, en especial en el área de gestión de la información, constituye una herramienta significativa a la hora de incrementar la participación e interacción por parte de los estudiantes. Permitió que algunas personas que en actividades más teóricas planteadas se posicionaron en una actitud pasiva, en el análisis de los casos, especialmente en el trabajo práctico descripto, se mostraran con un espíritu dinámico y creativo. Asimismo, la utilización de este método facilitó la conformación de un 


\section{Rebecin \\ EDUCAÇÃ̃O em CIÊNCIA da INFORMAÇÃO}

clima de trabajo adecuado para el desarrollo de pensamiento crítico en relación a posibles situaciones reales que deban afrontarse y abrió espacios de discusión tendientes a proponer diversos puntos de vista.

La articulación con la bibliografía propuesta es necesaria, así como con los contenidos expuestos en las clases tanto teóricas como prácticas. Aunque, cabe resaltar, se evita la repetición de conceptos o planteos teóricos y se invita a realizar el esfuerzo de articular con un escenario de gestión situado y de necesidad de toma de decisiones o acciones a emprender en tiempos limitados.

Con esta reflexión sobre el método de caso como estrategia didáctica y su aplicación concreta a una asignatura de la carrera de bibliotecología, se pretende compartir una experiencia que ojalá sea fructífera y de interés para quienes se enrolan en la compleja tarea de enseñar en el ámbito universitario.

\section{REFERENCIAS}

ARGANDOÑA, A. La enseñanza de la ética por el método de caso: 1999. In: FLECHA, J. R. Europa: ¿mercado o comunidad? De la Escuela de Salamanca a la Europa del futuro.
Salamanca: Publicaciones Universidad Pontificia, 1999.

BONACHE, J. El estudio de casos como estrategia de construcción teórica: características, críticas y defensas. Cuadernos de Economía y Dirección de la Empresa, Madrid, n.3, p.123-140, 1999.

CÉSAR, A. M. R. V. C. Método do estudo de caso (case studies) ou método do caso (teaching cases)? Uma análise dos dois métodos no ensino e pesquisa em administração. Revista Eletrônica Mackenzie de Casos, São Paulo, v.1, n.1, jul./dez. 2005. Disponível em:

<http://www.mackenzie.br/fileadmin/Gra duacao/CCSA/remac/jul_dez_05/06.pdf >. Acesso em: 29 mayo. $201 \overline{7}$.

CORDA, M. C. Enseñanza de la bibliotecología en la UNLP: la perspectiva en las asignaturas "Gestión de la Información" y "Usuarios de Información" de la Licenciatura/Profesorado en Bibliotecología y Ciencias de la Información. In: ENCUENTRO DE DIRECTORES Y VIII DE DOCENTES DE ESCUELAS DE BIBLIOTECOLOGÍA Y CIENCIA DE LA INFORMACIÓN DEL MERCOSUR, 9., 2012, Montevideo, Uruguay.

ESTRADA CUZCANO, A.; ALFANO MENDIVES, K. L. El método de casos como alternativa pedagógica para la enseñanza de la Bibliotecología y las Ciencias de la Información. Investigación Bibliotecológica, Ciudad de México, v.29, n.65, p.195212, jan./abr. 2015. Disponível em: <http://www.scielo.org.mx/pdf/ib/v29n65 /v29n65a9.pdf>. Acesso em: 29 mayo. 2017. 


\section{Rebecin \\ EDUCAÇÃO em CIÊNCIA da INFORMAÇÃO}

GARCÍA DEL JUNCO, J.;

CASTELLANOS VERDUGO, M. El método del caso y de las situaciones:

herramientas de diagnóstico y de decisión. Dirección y Organización, Madrid, n.19, p.96-117, jan. 1998.

Disponível em:

<http://www.revistadyo.es/index.php/dy o/article/view/330/330 > . Acesso em: 29 mayo. 2017.

GONZÁLEZ PÉREZ, E. Estudio de casos como estrategia didáctica en la formación del estudiantado en bibliotecología. e-Ciencias de la Información, San José (Costa Rica), v.5, n.2, p.1-13, jul./dez. 2015.

Disponível em:

$<$ http://revistas.ucr.ac.cr/index.php/ecie ncias/article/view/19736>. Acesso em: 29 mayo. 2017.

LAUDANO, C. N.; CORDA, M. C.; PLANAS, J. A. La cuestión tecnológica en los planes de estudio de la carrera de bibliotecología de la Universidad Nacional de La Plata. Aproximaciones críticas desde los documentos y la mirada de los actores. Información, Cultura y Sociedad, Buenos Aires, v.24, p.69-86, 2011. Disponível em: $<$ http://revistascientificas.filo.uba.ar/ind ex.php/ICS/article/download/735/712>. Acesso em: 11 abr. 2017.

MIGLIOLI, S. O método do caso aplicado ao ensino da Biblioteconomia: histórico e perspectivas. Encontros
Bibli: Revista Eletrônica de Biblioteconomia e Ciência da Informação, Florianópolis, v.19, n.39, p.1-18, jan./abr. 2014. Disponível em: <https://periodicos.ufsc.br/index.php/eb/ article/download/15182924.2014v19n39p1/26575>. Acesso em: 11 abr. 2017.

OGLIASTRI, E. El método de casos. Cali: Centro de Recursos para la Enseñanza y el Aprendizaje, 2005.

UNIVERSIDAD NACIONAL DE LA PLATA. FACULTAD DE HUMANIDADES Y CIENCIAS DE LA EDUCACIÓN. Plan de estudios de la carrera de Bibliotecología 2004. La Plata: UNLP, 2004. Disponível em: <http://www.memoria.fahce.unlp.edu.ar/ planes/pl.39/pl.39.pdf>. Acesso em: 11 abr. 2017.

VARGAS VASSEROT, C. EI método del caso en la enseñanza del derecho: experiencia piloto de un piloto novel. Revista de Formación e Innovación Educativa Universitaria, Vigo, v.2, n.4, p.193-206, 2009. Disponível em: $<$ http://refiedu.webs.uvigo.es/Refiedu/V ol2_4/REFIEDU_2_4_2.pdf>. Acesso em: 11 jun. $201 \overline{ }$.

WASSERMANN, S. Los casos como instrumentos educativos. In: WASSERMANN, S. El estudio de casos como método de enseñanza. Buenos Aires: Amorrortu, 2006. p.1731. 


\section{María Cecilia Corda}

Facultad Latinoamericana de Ciencias Sociales FLACSO Sede Argentina

Instituto de Investigaciones en Humanidades y Ciencias Sociales (UNLP-CONICET)

Facultad de Humanidades y Ciencias de la Educación (FaHCE)

Universidad Nacional de La Plata (UNLP)

E-mail: mccorda2003@yahoo.com.ar

Argentina

\section{Marcela Karina Coria}

Instituto de Investigaciones en Humanidades y Ciencias Sociales (UNLP-CONICET)

Facultad de Humanidades y Ciencias de la Educación (FaHCE)

Universidad Nacional de La Plata (UNLP)

E-mail: coria.marcela05@gmail.com

Argentina

\section{Ana Ayala}

Departamento de Bibliotecología

Facultad de Humanidades y Ciencias de la Educación (FaHCE)

Universidad Nacional de La Plata (UNLP)

E-mail: ayalaana1989@gmail.com

Argentina

\section{Rafaela Stefanizzi}

Departamento de Bibliotecología

Facultad de Humanidades y Ciencias de la Educación (FaHCE)

Universidad Nacional de La Plata (UNLP)

E-mail: rafaelastefanizzi@hotmail.com

Argentina 